\title{
Capture and sorting of multiple cells by polarization-controlled three-beam interference
}

\author{
Yu Hou ${ }^{1,2}$, Zuobin Wang ${ }^{1,2}$, Yaowei Hu${ }^{1}$, Dayou $\mathbf{L i}^{1,2}$ and Renxi Qiu ${ }^{2}$ \\ Q1 ${ }^{1}$ Changchun University of Science and Technology, JR3CN \& CNM, Changchun, 130022, People's \\ Republic of China \\ ${ }^{2}$ University of Bedfordshire, JR3CN \& IRAC, Luton, LU1 3JU, UK
}

Received 21 July 2015, revised 2 November 2015

Accepted for publication 5 November 2015

Published DD MM 2016

\begin{abstract}
For the capture and sorting of multiple cells, a sensitive and highly efficient polarizationcontrolled three-beam interference set-up has been developed. With the theory of superposition of three beams, simulations on the influence of polarization angle upon the intensity distribution and the laser gradient force change with different polarization angles have been carried out. By controlling the polarization angle of the beams, various intensity distributions and different sizes of dots are obtained. We have experimentally observed multiple optical tweezers and the sorting of cells with different polarization angles, which are in accordance with the theoretical analysis. The experimental results have shown that the polarization angle affects the shapes and feature sizes of the interference patterns and the trapping force.
\end{abstract}

Keywords: polarization-controlled, interferometric capture and sorting, optical tweezers

(Some figures may appear in colour only in the online journal)

\section{Introduction}

In recent years, optical technologies have been actively developed to play an important role in bioscience, especially research in the targeted drug delivery [1], trapping of macromolecules such as RNA and DNA [2, 3], and living cellular adhesion measurement [4]. Moreover, in the research field of biophysics, the particular type cells usually exist at the mixture of several different morphologies of samples, which need to be isolated and purified for further observation and analysis, such as the recent research of Cytotoxic T lymphocytes (CTLs) to find and kill cancer cells [5]. How to find CTLs and control them close to the cancer cells are both important for the work. Hence, the optical trapping has been presented with the features of remote manipulation and less damaging.

In contrast to the current experimental methods based on one beam optical trap that just manipulates one particle in one operation, there is a need to trap a number of particles simultaneously using the basic theory of optical tweezers. Some techniques to create spatially distributed multiple optical traps on various applications have been reported such as the spatial light modulator (SLM) which uses computer generated diffraction patterns [6], fast laser-scanning which is usually created by time-sharing of a single laser beam [7, 8], and multiple light sources which are usually generated by diffractive elements or laser beam interference (LBI) [9]. The most common approaches so far are those of using the SLM based on the high numerical aperture objectives to create computer-generated holographic optical traps, which could independently trap micro/nano particles in 3D. However, such methods are limited to manipulate small number particles in real-time due to their complex and expensive constructions, and prevent the multiple optical traps from being used as the general research instrument.

LBI is a promising technology for the volume creation of a large regular array of optical traps with easily constructed and inexpensive lenses. This method does not require strongly focused beams and provides a way to generate periodical and quasi-periodical optical traps with adjustable periods and less damaging, so that simultaneous spatial manipulation of a large number of particles can be achieved [10]. However, compared with the current approaches, most of studies for the creation of multiple optical traps are limited to only create a pattern of linear interference fringes or a few of dot traps 
without the adjustment of the formation. Furthermore, the trapping force is only adjusted by the changing of laser intensity and it is not stable due to the output variations.

In this work, an adjustable three-beam interference method was presented. The different sizes of cells could be manipulated by the adjustable periodic structures which focus on the yeast suspensions. We demonstrated the trapping of yeast cells in an interference array of dots with regular triangle structures, and provided the force analysis of yeast cells in the laser interference fields. From the real-time visual feedback by CCD, the different sizes of cells were manipulated by laser interference with different polarization angles in the same case.

\section{Interaction of the periodic structures of gradient laser field with yeast cells}

\subsection{Generation of the periodic structures of gradient laser field}

In this work, three-beam laser interference was used to generate laser interference patterns in the sample plane. The theoretical equations based on the three-beam laser interference are derived with proposed polarization modes. A laser interference pattern can be seen as the superposition of the electric field vectors when two or more coherent beams focus on one point. The electric field vector $E_{n}$ of a coherent beam can be expressed as [11]

$$
E_{n}=A_{n} p_{n} \exp \left[\mathrm{i}\left(k_{n} \cdot r-\omega t+\delta_{n}\right)\right],
$$

where $A_{n}$ is the beam amplitude, $p_{n}$ is the polarization vector, $k_{n}$ is the wave number, $r$ is the position vector. $\omega$ is the angular frequency, and $\delta_{n}$ is the initial phase. In the adjustable three-beam interference experimental setup, the three beams are allowed to form a symmetrical configuration with the same incident angles and the azimuthal angle $\varphi_{n}$ is fixed as $0^{\circ}, 120^{\circ}$ and $240^{\circ}$, respectively. Thus, $p_{n}$ and $k_{n}(n=1,2,3)$ can be expressed as

$$
\left\{\begin{aligned}
& p_{1}=-\left(\cos \theta \cos \psi_{1}\right) \cdot x-\sin \psi_{1} \\
& \cdot y-\left(\sin \theta \cos \psi_{1}\right) \cdot z \\
& p_{2}=\left(\frac{1}{2} \cos \theta \cos \psi_{2}+\frac{\sqrt{3}}{2} \sin \psi_{2}\right) \\
& \cdot x-\left(\frac{\sqrt{3}}{2} \cos \theta \cos \psi_{2}-\frac{1}{2} \sin \psi_{2}\right) \\
& \cdot y-\left(\sin \theta \cos \psi_{2}\right) \cdot z \\
& p_{3}=\left(\frac{1}{2} \cos \theta \cos \psi_{3}-\frac{\sqrt{3}}{2} \sin \psi_{3}\right) \\
& \cdot x+\left(\frac{\sqrt{3}}{2} \cos \theta \cos \psi_{3}+\frac{1}{2} \sin \psi_{3}\right) \\
& \cdot y-\left(\sin \theta \cos \psi_{3}\right) \cdot z \\
& k\left(\sin \theta \cos \varphi_{n} \cdot x+\sin \theta \sin \varphi_{n} \cdot y-\cos \theta \cdot z\right)
\end{aligned}\right.
$$

where $x, y$ and $z$ are the unit vectors in the three directions of coordinates. $\theta_{n}$ is the incident angle, $\psi_{n}$ is the polarization angle, and $k=2 \pi / \lambda$ is the wave number, $\lambda$ is the wavelength of light.

According to equations (1) and (2), the intensity distribution of three-beam interference can be expressed as

$$
\begin{aligned}
I(r) & =\sum_{n=1}^{3} \sum_{m=1}^{3}\left|E_{n}\right|\left|E_{m}\right| \cos \left\langle E_{n} \cdot E_{m}\right\rangle \\
& =\sum_{n=1}^{3} \sum_{m=1}^{3} A_{n} p_{n} \cdot A_{m} p_{m} \cdot \mathrm{e}^{\left[\mathrm{i}\left(k_{n}-k_{m}\right) r\right]}
\end{aligned}
$$

with the spatial period of the 2D hexagonal structure [11]

$$
d_{0}=\frac{2 \lambda}{3 n_{m} \sin \theta},
$$

where $n_{m}$ is the refractive index of the surrounding medium.

The distributions of interference patterns generated by computer simulation with different polarization modes are shown in figure 1 . The intensity distributions of interference patterns can be changed by the polarization mode, such as regular triangle distributions, elliptical distributions and circular distributions. Compared with other multi-beam interference methods, three-beam interference has the advantage of generating no modulation which can be easily controlled in the interference field. Hence, the polarization angle becomes a potential parameter for modulating the intensity of the interfering field. By changing the polarization angle of three interference beams, various intensity distributions are obtained, as shown in figure 1.

\subsection{Gradient force in the interference field}

In the previous work, it demonstrated that only the gradient force existed in the transverse direction without any component of the light pressure force when the standing wave acted on a particle [12]. The gradient force could originate from the interaction of the light-induced dipole moment between the electrical component of light and the particle [13]. In this section, we discuss the influence and the orientation of gradient when the particle is placed in the threebeam interference field, and the sample used is considered as a spherical particle. According to the report by Rohrbach et al the polarizability of particle can decide the strength of both forces, and only the gradient force acts on the particles, when they are placed into the interference field, and it will move towards the maximum intensity of the periodic interference structures [14].

As shown in figure 2, it is the typical transparent particle assumed to be located close to the central axis of a stream of photons. On the surface of the particle, part of the photons will be reflected and the other will be refracted which are deviated from the initial direction and incident onto the upper surface of the particle. Then the photons will continue being reflected and refracted in the next location. Hence, due to the reflected or transmitted photons at the lower or upper surface 


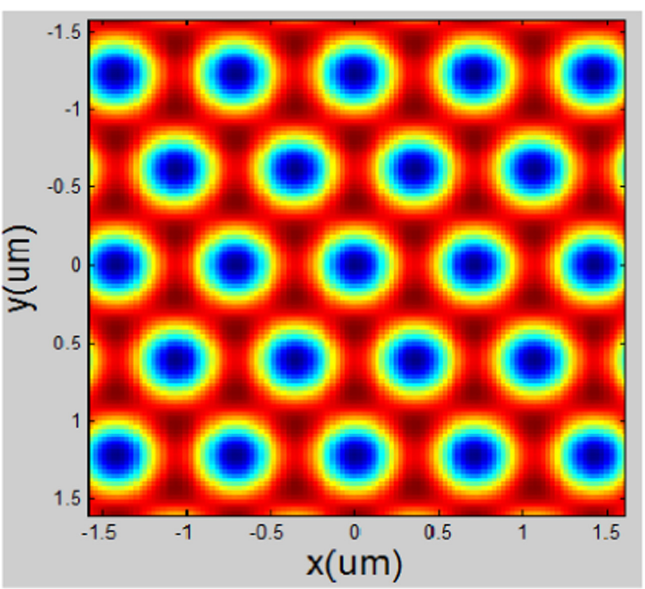

(a)

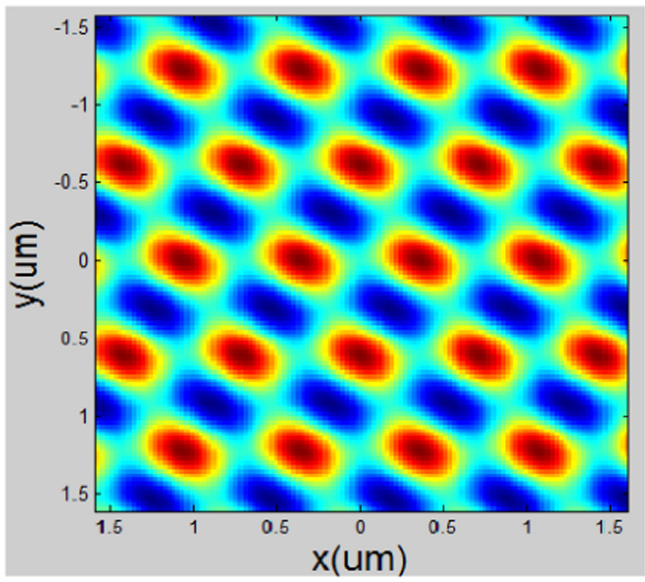

(c)

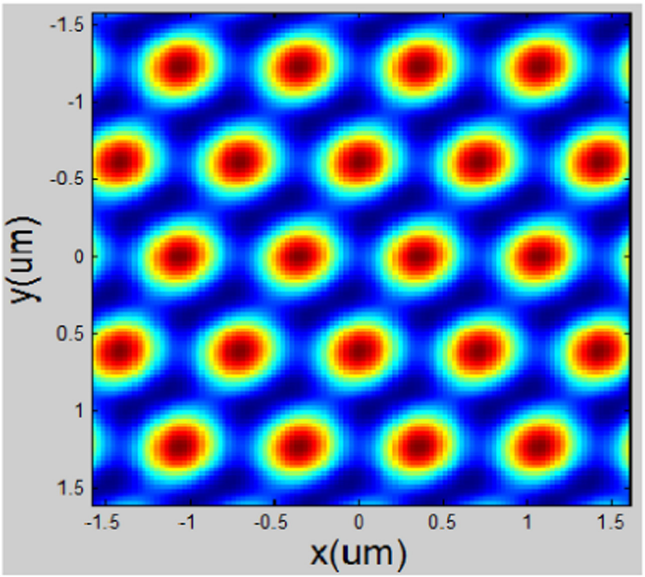

(e)

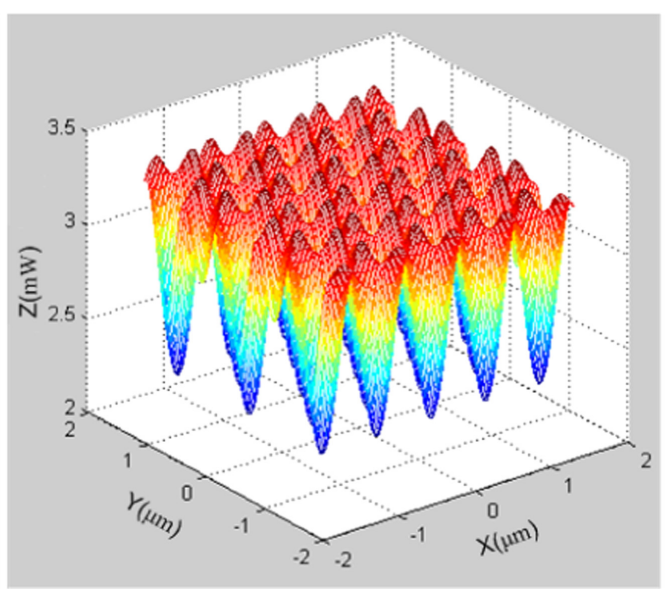

(b)

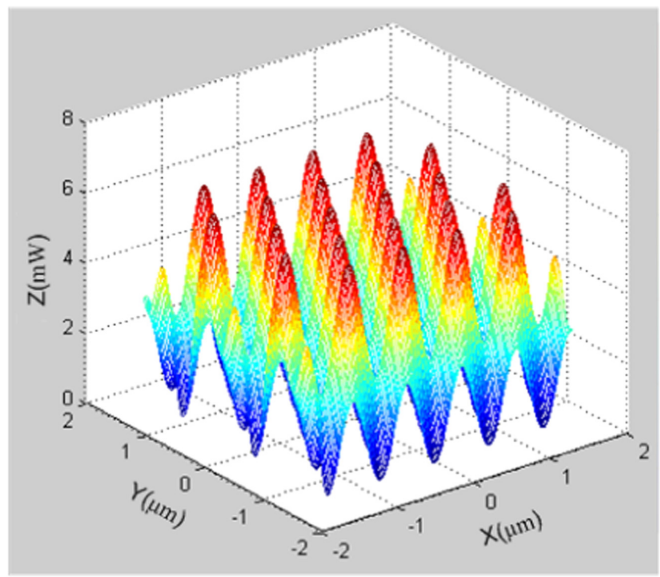

(d)

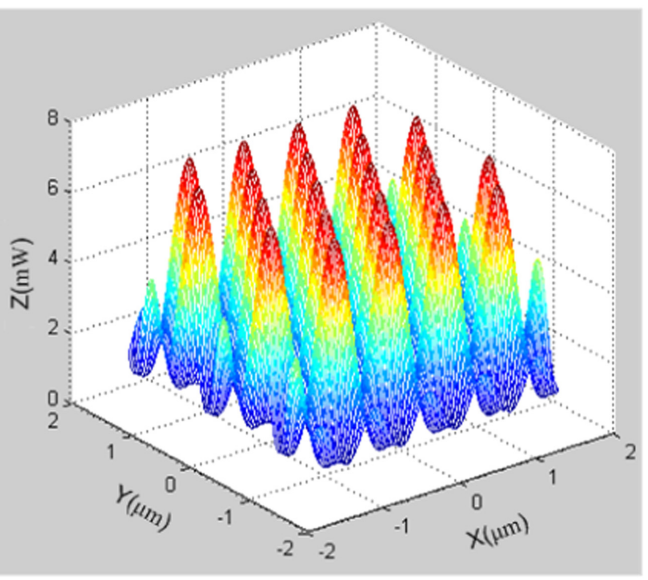

$(f)$

Figure 1. Computer simulations of laser interference. (a), (c) and (e) 2D intensity distributions for three-beam interference with the polarization modes of TE-TE-TE, TE-TM-TM and TE-TE-TM, respectively. (b), (d) and (f) 3D intensity distributions for three-beam interference with the polarization mode relative to (a), (c) and (e), respectively $\left(\lambda=532 \mathrm{~nm}, \theta=30^{\circ}\right)$. 


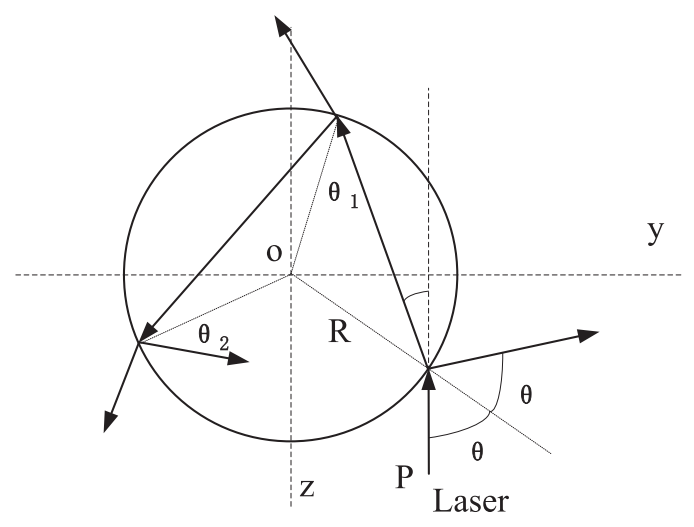

Figure 2. Schematic of the laser incident upon the lower side of the particle [15].

of the particle, the gradient force can be expressed as [15]

$$
\begin{aligned}
F_{\text {grad }}= & -\int_{0}^{\pi / 2} \int_{0}^{2 \pi} I(\rho, z) \frac{n_{0}}{2 c} \sin (2 \theta)\left|r_{1}\right|^{2} \\
& \times r^{2} \cos (\phi) \times \sin (2 \theta) \mathrm{d} \phi \mathrm{d} \theta \\
& +\int_{0}^{\pi / 2} \int_{0}^{2 \pi} I(\rho, z) \frac{n_{s}}{2 c} \sin \left(\theta-\theta_{1}\right)\left|t_{1}\right|^{2} \\
& \times r^{2} \cos (\phi) \times \sin (2 \theta) \mathrm{d} \phi \mathrm{d} \theta \\
& +\int_{0}^{\pi / 2} \int_{0}^{2 \pi} I(\rho, z) \frac{n_{s}}{2 c}\left[\sin \left(3 \theta_{2}-\theta\right)\right. \\
& \left.-\sin \left(\theta-\theta_{1}\right)\right] \times\left|t_{1}\right|^{2}\left|r_{1}\right|^{2} \\
& \times r^{2} \cos (\phi) \sin (2 \theta) \mathrm{d} \phi \mathrm{d} \theta \\
& +\int_{0}^{\pi / 2} \int_{0}^{2 \pi} \frac{I(\rho, z)}{2 c}\left\{n_{0} \sin \left[2\left(\theta-\theta_{1}\right)\right]\right. \\
& \left.-n_{s} \sin \left(\theta-\theta_{1}\right)\right\}\left|t_{1}\right|^{2}\left|t_{1}\right|^{2} \\
& \times r^{2} \cos (\phi) \sin (2 \theta) \mathrm{d} \phi \mathrm{d} \theta,
\end{aligned}
$$

where $n_{s}$ is the refraction index of the particle, $n_{0}$ is the refraction index of the surround, $r$ is the radius of the particle and $c$ is the speed of the laser in the surround. In the equation, the power reflectance for the lower surface of the particle can be written as [16]

$$
\begin{aligned}
\left|r_{1}\right|^{2}= & \left(\left(n_{0} n_{s}\right)^{2}\left[\cos ^{2}(\theta)-\cos ^{2}\left(\theta_{1}\right)\right]^{2}\right) / \\
& \left(\left\{n_{0} n_{s}\left[\cos ^{2}(\theta)+\cos ^{2}\left(\theta_{1}\right)\right]\right.\right. \\
& \left.\left.\left.+\left(n_{\mathrm{o}}^{2}+n_{s}^{2}\right) \cos (\theta) \cos \left(\theta_{1}\right)\right\}^{2}\right)\right) .
\end{aligned}
$$

Moreover, the power transmittance can be written as

$$
\left|t_{1}\right|^{2}=1-\left|r_{1}\right|^{2}
$$

The intensity profile of the beam $I(\rho, z)$ can be written as the lowest-order Gaussian mode [16]

$$
I(\rho, z)=\frac{2 P}{\pi W^{2}} \exp \left[\frac{-2 \rho^{2}}{W^{2}}\right],
$$

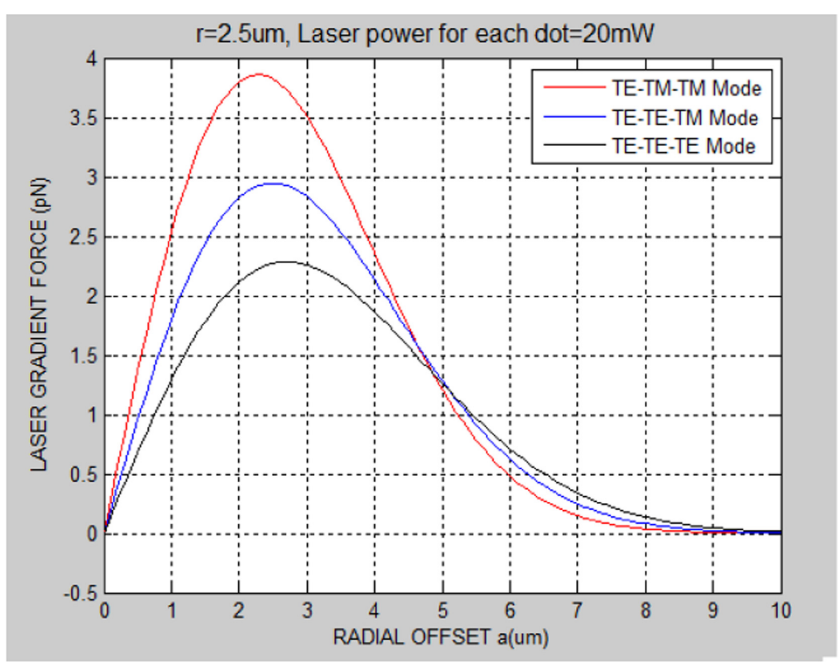

Figure 3. Laser gradient force versus the radial offset. The cell located in the interference field experiences a centering force pulling it into the alignment with the central axis of the dot.

where $\rho$ is the gradient distance from the axis of the laser. $W$ is the width of the laser beam and given by [15]

$$
W=W_{0}\left[1+\left(\frac{z}{z_{0}}\right)^{2}\right]^{1 / 2},
$$

where $z$ and $z_{0}$ are the distances measured along the beam's direction of propagation with $z=0$ and $I(\rho, z)=I_{\max } / 2$, respectively.

Hence, the TE-TE-TE mode, TE-TE-TM mode and TE-TM-TM mode have been programmed in Matlab with equation (5) for the numerical computation purposes. Each dot in the interference field was used as one incident beam in the simulation. Figure 3 shows the force in a sphere cell as a function of the interaction between the dot center and the central axis of cell. The radial offset $a$ is the relative offset between the beam center and the central axis of cell. $W_{0}$ is the width of each dot in the three-beam interference pattern. The parameters for the particle and the beam were chosen as $n_{s}=1.5468$ and $n_{0}=1.333$. The laser power for each dot was $20 \mathrm{~mW}$, the wavelength of the laser was $532 \mathrm{~nm}$ and the radius of the particle was $2.5 \mu \mathrm{m}$.

As shown in figure 3, the laser gradient force acted on the cells can be changed by the different sizes of dots in the interference field. The comparison of the laser gradient force curves with different widths of dots shows that the gradient force can be much larger when the size of cells is approximately equal to $W_{0}$. The gradient forces also act over a smaller range around the dot center. Moreover, the trapping force will start falling when the offset is greater than $3 \mu \mathrm{m}$ which has almost the same conclusion as that in section 4.1 . Thus, the neighboring optical trap would not influence the particle due to the rapidly decreased trapping force. 


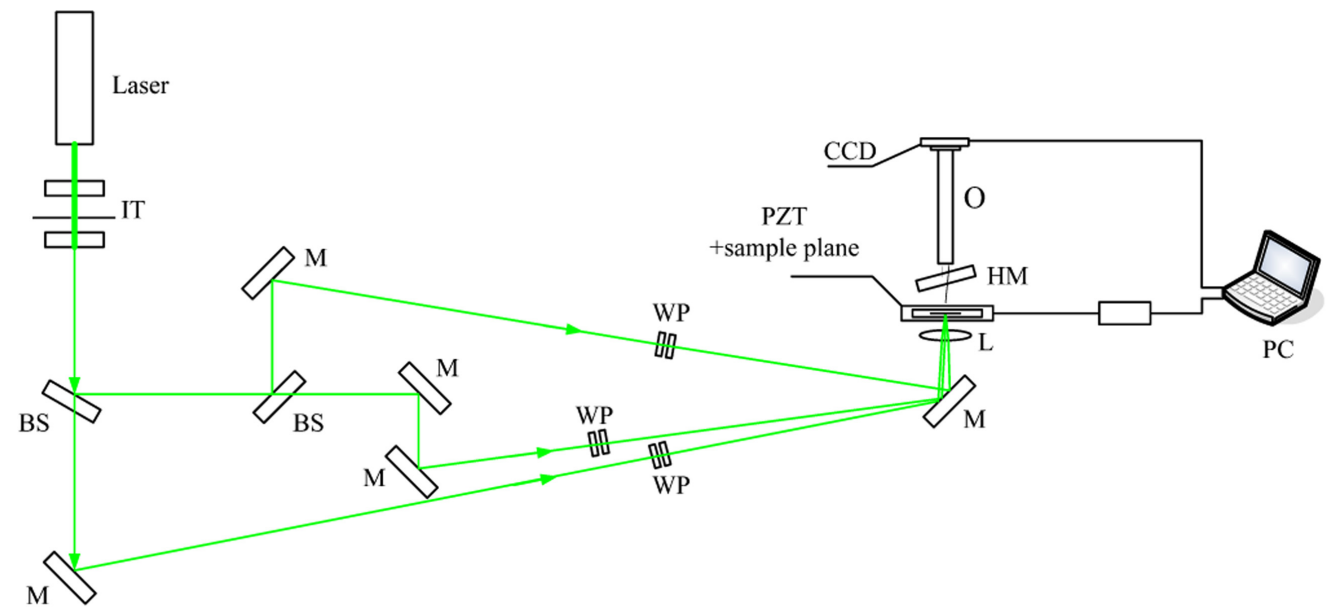

Figure 4. Polarization-controlled three-beam interference experimental set-up. The three interfering beams are placed symmetrically around the $z$-axis with the angle $\theta$. IT, inverted telescope; M, mirror; BS, beam-splitter; WP, half-wave plate and polarization prism; L, lens; O, microscope; CCD, charge-coupled device; PZT, piezoelectric transducer; PC, personal computer.

\section{Experimental setup}

The polarization-controlled three-beam interference experimental set-up is shown in figure 4. It is essentially based on two blocks: an adjustable three-beam interference system for producing the periodic structure and a real-time visual feedback system for imaging and monitoring the movement of cells. A semiconductor continuous laser made by CNI (Changchun New Industries Optoelectronics Technology Co., Ltd), operating in the $\mathrm{TEM}_{00}$ mode at $532 \mathrm{~nm}$ and with the output power from 0 to $2 \mathrm{~W}$, was used as a light source to provide both the distinct periodic structure in the visible spectral range with a longer coherence length and the necessary gradient force by the intensity. Firstly, due to the same non-uniformity distribution of periodic structures caused by the non-uniform Gaussian beam interference, an inverted Kaplerian telescope system with two positive focus lenses having different focal lengths and a $50 \mu \mathrm{m}$ pinhole was used. The focal length of $f_{1}$ was larger than that of $f_{2}$. In the experiment, the $f_{1}=100 \mathrm{~mm}$ and $f_{2}=35 \mathrm{~mm}$ lenses were used, and the magnification was 1/2.85 times. Moreover, due to the existence of intensity variation in the input Gaussian beam, a diameter of $50 \mu \mathrm{m}$ pinhole was used in the experiment to improve the spatial quality of the beam. The emerging beam from the alignment and filtering system has a diameter close to $1 \mathrm{~mm}$ and was split by two $50: 50$ beam splitters (BS) into three beams and focused by the $10 \mathrm{~cm}$ focal length lens $L_{1}$ on the sample plane. The interference pattern in the sample plane was formed into about $100 \mu \mathrm{m}$ spots in space. Meanwhile, a 3D piezoelectric ceramic transducer (PZT) controller together with the sample stage was used to operate the sample moving on a flat surface and make the sample matching to the three-beam focus. The observation part consisted of a flat mirror HM which could totally reflect $532 \mathrm{~nm}$ laser, and the microscope $\mathrm{O}$ for imaging the sample plane on a CCD camera. Finally, the images were transmitted to the computer. Three polarizers were chosen as the modulation devices to change the output energy and the polarization angles. The various interference patterns were formed by different combinations of polarization angles when they had the same incident angles. According to the real-time visual feedback by the CCD camera, the computer was able to obtain the information of sample cells. Then, the matching interference pattern was adjusted by changing the laser polarization angles.

\section{Results and discussions}

\subsection{Optical trapping and manipulation of yeast cells}

The yeast cells with spherical shapes were used in the experiment. All of pelleted cells were grown for $24 \mathrm{~h}$. An aliquot of sedimented yeast amount was diluted in 10 times with the deionized water without stirring. The yeast suspensions were placed on a non-coated cover-slip when the cells were captured by the adjustable three-beam interference experimental setup described above. The period of interference pattern was chosen slightly larger than the average diameter of yeast cells, and the laser intensity at the center of the interference pattern was about $950 \mathrm{~mW}$. We observed the multiple yeast cells captured by the laser gradient force originated from the interaction of light-induced dipole moment. Figure 5 shows that the optical trapping of tens of yeast cells in the interference field illuminated by three-beam interference with the TE-TE-TM mode was achieved. With the laser gradient forces, the whole array of optically trapped yeast cells could be dragged by the movement of PZT.

According to the Stokes' theorem, the maximum deviated speed of yeast cells oriented from the center of each dot, formed by three-beam interference, could be calculated by [17]

$$
F_{\text {drag }}=6 \pi \eta R v \text {. }
$$

where $\eta$ is the viscosity of the fluid surrounding the cells and $R$ is the cell radius. The yeast cells were deviated by the maximum stokes force and settled in a new equilibrium 


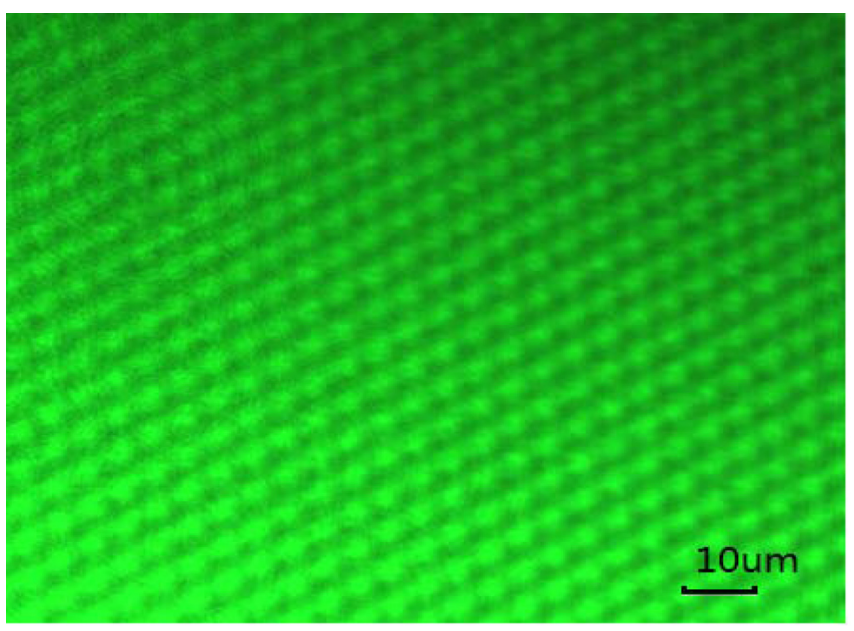

(a)

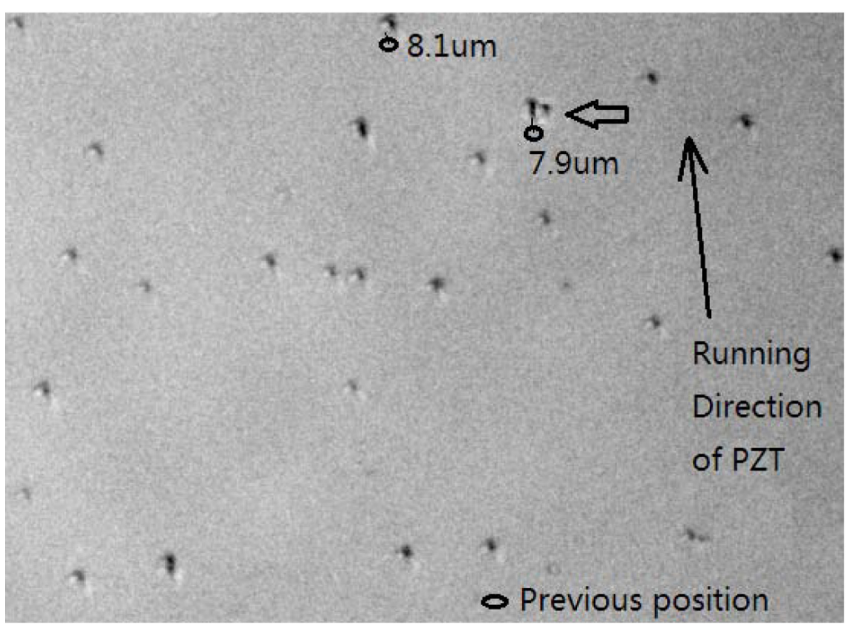

(c)

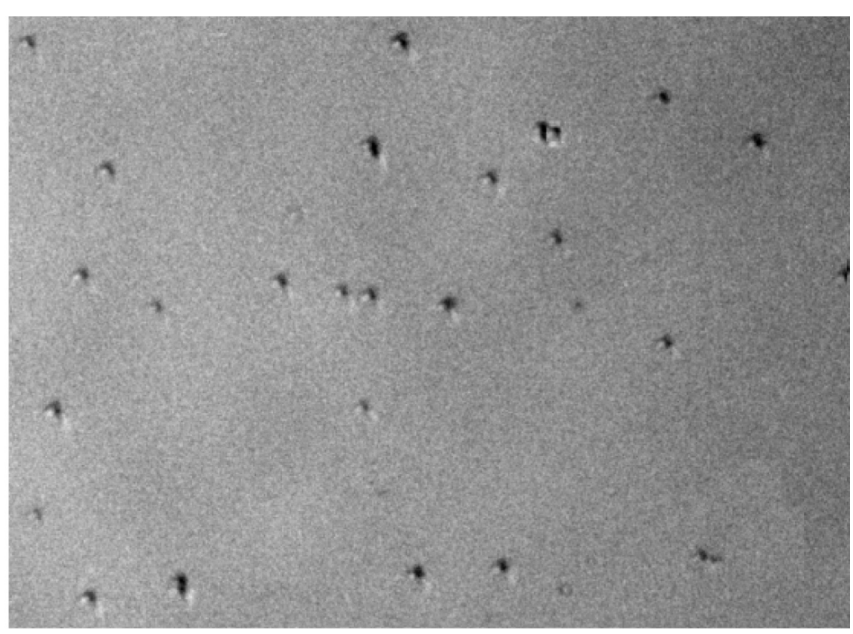

(b)

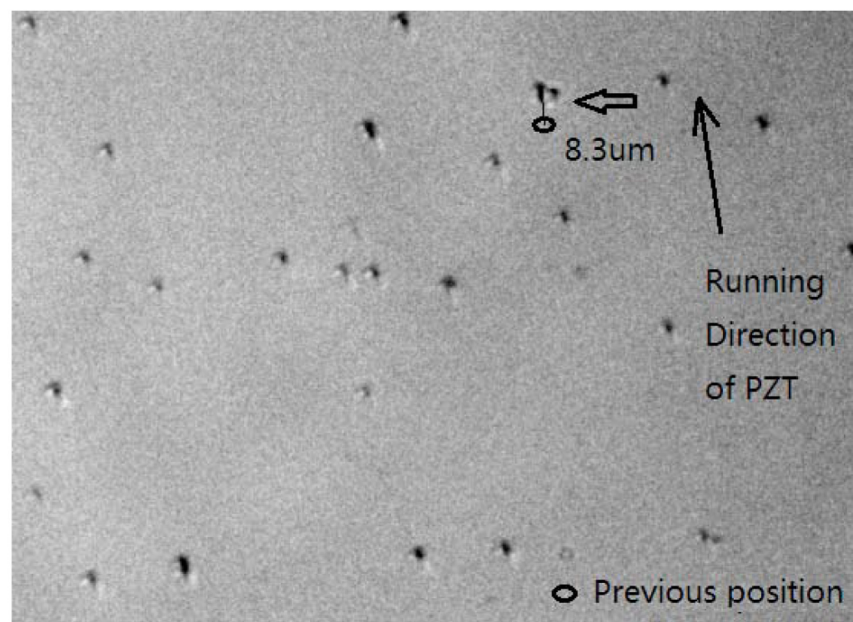

(d)

Figure 5. Spatial distributions of the yeast cells in the interference field. (a) Experimentally obtained patterns generated on the sample plane. (b) Initial spatial distribution of yeast cells in the sample plane in the case where the interference was switched off, and the yeast cells were in equilibrium. (c) The yeast cells were moved at a velocity of about $2 \mu \mathrm{m} \mathrm{s}^{-1}$ in the case where the interference was switched off. (d) The yeast cells were moved at a velocity of about $2 \mu \mathrm{m} \mathrm{s}^{-1}$ in the case where the interference was switched on.

location where the drag force was compensated by the laser gradient force in the opposite direction. Figure 5 demonstrates this effect for yeast cells. Figure 5(a) shows a picture of regular triangle distribution of dots in an interference pattern observed by CCD. Thus, according to equation (5), the incidence angle is about $4^{\circ}$, and the period of the $2 \mathrm{D}$ hexagonal lattice array is about $5 \mu \mathrm{m}$. Figure $5(\mathrm{~b})$ gives a picture of the initial spatial distribution of yeast cells and the yeast cells were in equilibrium. Figure 5(c) shows a picture of the movement of the yeast cells in the interference field where the interference was switched off. In this case, all of the yeast cells were dragged by the movement of PZT. Figure 5(d) shows the picture of the movement of the yeast cells in the interference field where the interference was switched on. Then, most of yeast cells were dragged by the laser gradient force except the bigger object indicated by the arrows in the figure which could be agglomerated cells. Figure 5(d) shows that the yeast cells were dragged at a velocity of about
$2 \mu \mathrm{m} \mathrm{s}^{-1}$, and then the small cells moved more rapidly towards the movement direction than large cells. Because the yeast cells are trapped by the multiple optical tweezers and the stage is dragged by the movement of PZT, the bigger object shows the relative movement of other yeast cells. According to the measurements, the interference trapping of yeast cells took place in the equilibrium at a steady rate as high as $11.6 \mu \mathrm{m} \mathrm{s}^{-1}$, and the trapping force started falling when the offset was greater than $1.5 \mu \mathrm{m}$.

\subsection{Optical sorting of yeast cells with different polarization angles}

Cell sorting is accomplished through the changing of laser gradient force which is proportional to the specific polarizability of the cells and the light intensity distribution in the interference field. The cell array was moved at a constant speed in the experiment, which avoided the effects of traditional manipulation with the trapezoidal acceleration motion. 


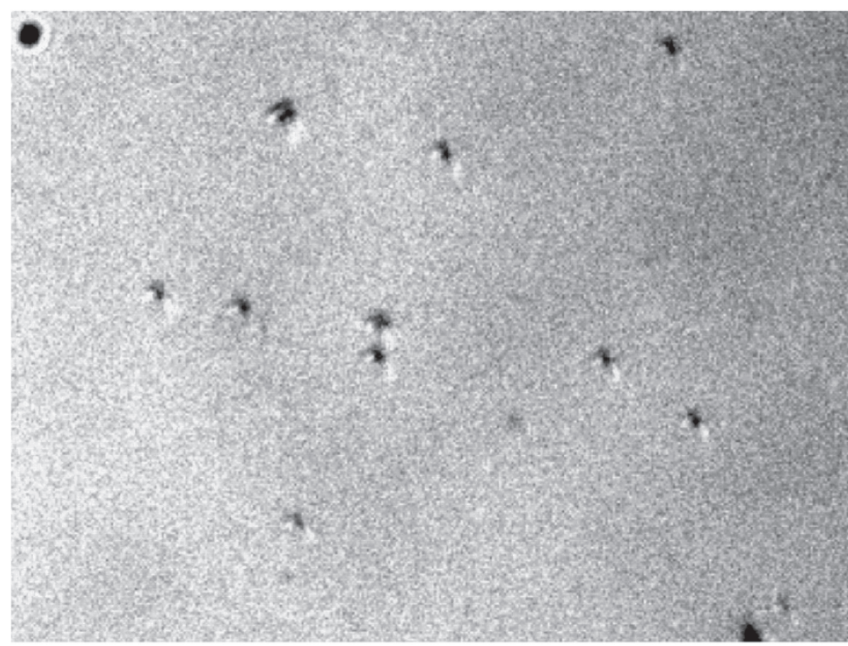

(a)

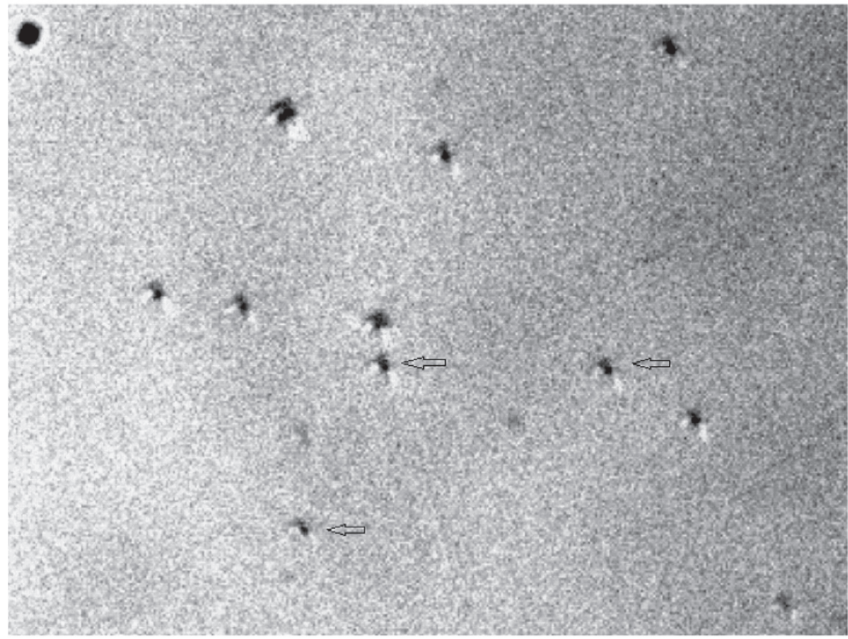

(c)

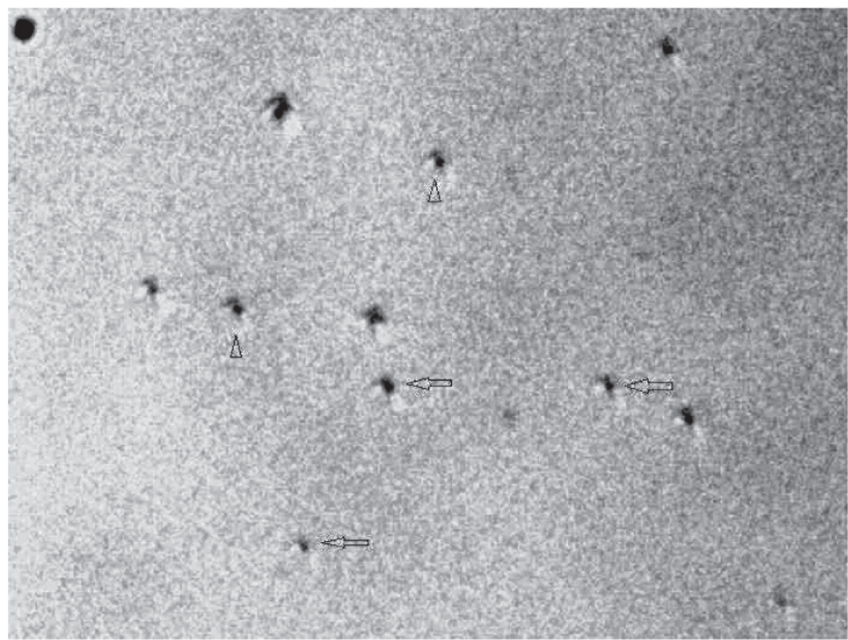

(e)

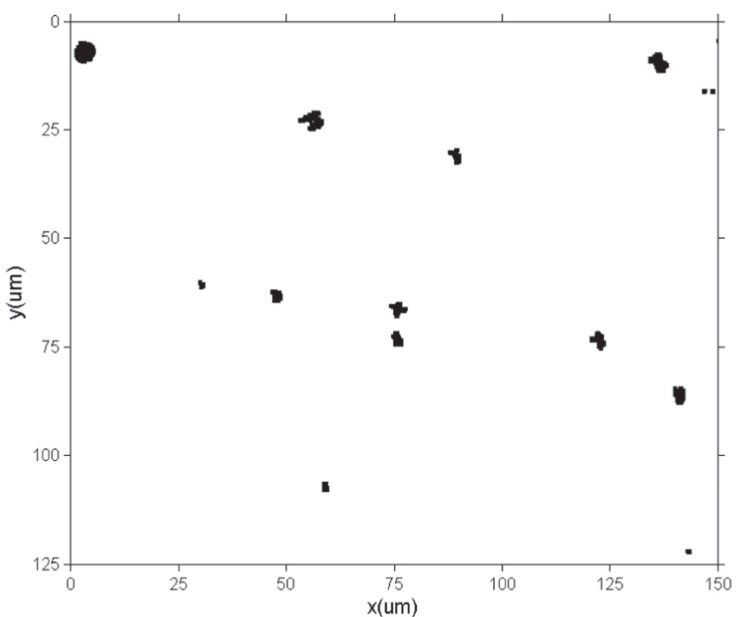

(b)

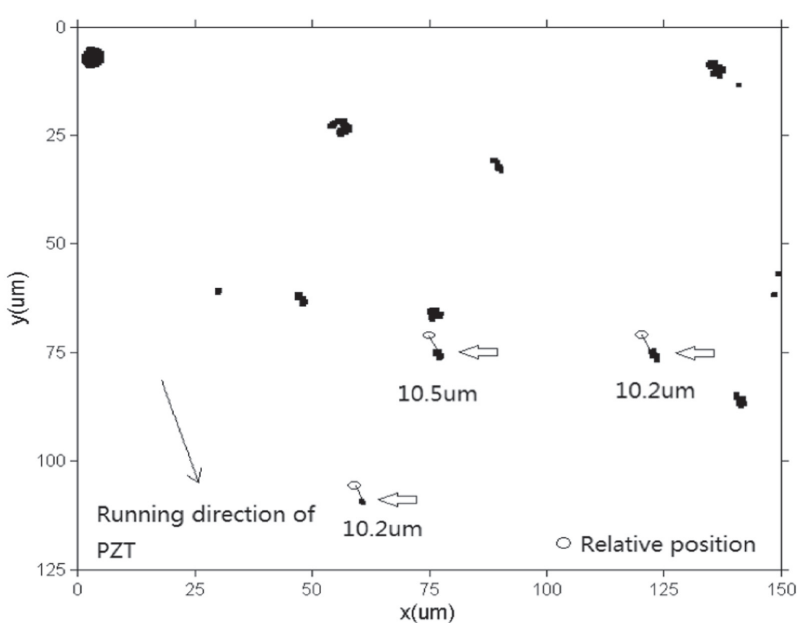

(d)

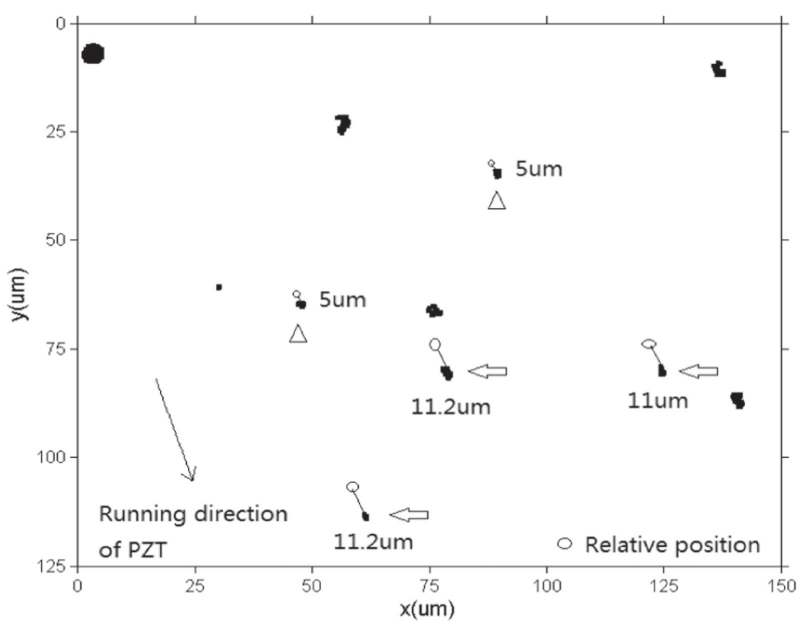

(f)

Figure 6. Sorting dynamics of the interested cells with different polarization modes. (a) Cell distribution in the illuminated field where the interference was switched off. (c) Cell distribution in the illuminated field where the interference was switched on with the TE-TE-TM mode. (e) Cell distribution in the illuminated field where the interference was switched on with the TE-TE-TE mode. (b), (d) and (f) The spatial relationships with each cell generated by MATLAB corresponding to (a), (c) and (e), respectively. 
Then, the light intensity distribution in the interference field could be redistributed with the change of polarization angle, and the cells of interest were captured from the trap array via the different drag forces. We also investigated the effect of optical sorting of yeast cells with different polarization angles.

The cells of interest could be isolated by a suitable Stokes force or drag speed corresponding to the laser gradient force. Only the cells close to a certain critical size could be captured and transported by a moving gradient force and also be in the equilibrium in the motion.

As shown in figure 6, PZT is moved at a constant speed, and the cells have been dragged in different interference fields. Figure 6 demonstrates the experimental implementation of the sorting of yeast cells when the distribution of interference field is changed. By controlling the polarization angles of beams, various intensity distributions and different sizes of dots were obtained. Figure 6(a) shows ten cells in the illuminated field in the case where the interference was switched off. In figure 6(b), the interference was switched on with the TE-TE-TM mode, and the interference field was moved by PZT at a sustained speed of $2.5 \mu \mathrm{m} \mathrm{s}^{-1}$. It can clearly be seen that there are three cells relatively moved toward the lower right, indicated by arrows, and the other seven cells were trapped by the laser interference field. Then, the polarization angle is changed from the TE-TE-TM mode to the TE-TE-TE mode. The dot width in the interference field is reduced to provide the laser gradient force which is smaller than that provided by the TE-TE-TM mode. As shown in figure 6(c), there are five cells relatively moved by the movement of PZT due to the change of dot size and laser intensity distribution which is in accordance with the conclusion from figure 1 . Moreover, two cells which are indicated by the triangles have been moved less than the three other cells due to the insufficient dragged force. With the change of the polarization angle, the shapes of the interference patterns and the value of maximum intensity change correspondingly. The simulation and the experiments have shown that the polarization angles of three interference beams have a significant impact on the shapes of the patterns and the laser gradient force.

Compared with other multi-beam interference optical tweezers, without changing the device alignment of the setup, a variable and stable laser gradient force can be produced by the polarization-controlled three-beam interference experimental set-up. The set-up can generate various periodic structures with different intensities and dot sizes. Moreover, manipulation at a constant speed can avoid the influence of PZT acceleration. The selective capture and sorting of cells of different sizes by controlling the polarization angles of three beams can have new interesting possibilities of applications in cytology. In particular, applications such as sorting and separation of CTLs from a mixture of cells, capture and transportation of multiple cells are expected. The capture and sorting of biological cells are two important issues in biophysical research, and the polarization-controlled three-beam interference method can provide a way to generate various intensity distributions of optical traps with easily adjustable periods.

\section{Conclusions}

In this work, we have demonstrated a method for the capture and sorting of hundreds of cell samples using a novel polarization-controlled three-beam interference method. The capture and sorting of yeast cells in the interference array of dots based on the change of polarization angle has been discussed. We have experimentally observed multiple optical tweezers and the sorting of cells with different polarization angles, which are in accordance with the theoretical analysis. The experimental results have shown that the polarization angle affects the shapes and feature sizes of the interference patterns and the trapping force. The proposed method has its unique potential in biological research, which will be useful for the capture and sorting of cells, and the manipulation of them for further analysis.

\section{Acknowledgments}

This work was supported by International Science and Technology Cooperation Program of China (No.2012DFA11070), EU FP7 (ECROBOT No.318971; BioRA No.612641), EU H2020 (FabSurfWAR No.644971), Doctoral Program of Higher Education of China (No.20112216110002), Changbai Scholar Program of Jilin Province, National Natural Science Foundation Program of China (Nos.61176002 and 11103047), Jilin Provincial Science and Technology Program (Nos.201215136 and 20140414009GH), and Science and Technology Program of Changchun City (No.11KP04).

\section{References}

[1] Xin H, Li X and Li B 2011 Massive photothermal trapping and migration of particles by a tapered optical fiber Opt. Express 19 17065-74

[2] McCauley M J and Williams M C 2007 Mechanisms of DNA binding determined in optical tweezers experiments Biopolymers 85 154-68

[3] Samantha F et al 2011 Raman spectroscopy of individual monocytes reveals that single-beam optical trapping of mononuclear cells occurs by their nucleus J. Opt. 13044021

[4] Castelain M et al 2012 Single-cell adhesion probed in situ using optical tweezers: a case study with saccharomyces cerevisiae J. Appl. Phys. 111 114701-13

[5] Ritter A T et al 2015 Actin depletion initiates events leading to granule secretion at the immunological synapse Immunity $\mathbf{4 2}$ 864-76

[6] Jákl P, Siler M and Zemánek P 2014 Experimental analysis of multiple-beam interference optical traps Polish-SlovakCzech Optical Conf. on Wave and Quantum Aspects of Contemporary Optics (International Society for Optics and Photonics) pp 944105-6

[7] Mirsaidov U et al 2008 Live cell lithography: using optical tweezers to create synthetic tissue Lab Chip 8 2174-81 
[8] Liu C X et al 2015 Manipulating ellipsoidal micro-particles by femtosecond vortex tweezers J. Opt. 17035402

[9] Ják1 P et al 2014 Optical sorting of nonspherical and living microobjects in moving interference structures Opt. Express 22 29746-60

[10] Kruchenok J V et al 2005 Orientation of red blood cells and rouleaux disaggregation in interference laser fields $\mathrm{J}$. Biol. Phys. 31 73-85

[11] Zhang $\mathrm{J}$ et al 2014 Effects of azimuthal angles on laser interference lithography Appl. Opt. 53 6294-301

[12] Rubinov A N 2003 Physical grounds for biological effect of laser radiation J. Phys. D: Appl. Phys. 36 2317-30
[13] Ashkin A et al 1986 Observation of a single-beam gradient force optical trap for dielectric particles Opt. Lett. 11 288-90

[14] Rohrbach A and Stelzer E 2001 Optical trapping of dielectric particles in arbitrary fields J. Opt. Soc. Am. A 18 839-53

[15] Gauthier R C and Wallace S 1995 Optical levitation of spheres: analytical development and numerical computations of the force equations J. Opt. Soc. Am. B 12 1680-6

[16] Saleh B E A and Teich M C 2007 Fundamentals of Photonics vol 45 (New York: Wiley) p 87 Spie Org.

[17] Casaburi A et al 2005 Two- and three-beam interferometric optical tweezers Opt. Commun. 251 393-404 


\section{QUERY FORM}

JOURNAL: Journal of Optics

AuthoR: Y Hou et al

TITLE: Capture and sorting of multiple cells by polarization-controlled three-beam interference

ARTICLE ID: joptaa09d9

The layout of this article has not yet been finalized. Therefore this proof may contain columns that are not fully balanced/ matched or overlapping text in inline equations; these issues will be resolved once the final corrections have been incorporated.

SQ1

Please be aware that the colour figures in this article will only appear in colour in the online version. If you require colour in the printed journal and have not previously arranged it, please contact the Production Editor now.

\section{Page 1}

Q1

Please specify the corresponding author and provide his/her email address. 